\title{
Bulk ionic screening lengths from extremely large-scale molecular dynamics simulations
}

\author{
Johannes Zeman, ${ }^{1}$ Svyatoslav Kondrat, ${ }^{2,3,4}$ and Christian Holm ${ }^{1, *}$ \\ ${ }^{1}$ Institute for Computational Physics, University of Stuttgart, D-70569 Stuttgart, Germany \\ ${ }^{2}$ Department of Complex Systems, Institute of Physical Chemistry, \\ Polish Academy of Sciences, 01-224 Warsaw, Poland \\ ${ }^{3}$ Max-Planck-Institut für Intelligente Systeme, \\ Heisenbergstraße 3, 70569 Stuttgart, Germany \\ ${ }^{4} I V$. Institut für Theoretische Physik, Universität Stuttgart, \\ Pfaffenwaldring 57, 70569 Stuttgart, Germany
}

Recent experiments have reported anomalously large screening lengths of interactions between charged surfaces confining concentrated electrolytes and ionic liquids. Termed underscreening, this effect was ascribed to bulk properties of dense ionic systems. Herein, we study bulk ionic screening with extremely large-scale molecular dynamics simulations, allowing us to assess the range of distances relevant to the experiments. Our results yield two screening lengths satisfying distinct scaling relations. However, with an accuracy of $10^{-5} k_{\mathrm{B}} T$ in interionic potentials of mean force, we find no signs of underscreening, suggesting that other than bulk effects might be at play in the experiments.

Concentrated electrolytes and room-temperature ionic liquids (ILs) are playing an increasingly important role in science and technology, with applications ranging from organic synthesis, catalysis and analytical chemistry to electrochemical energy storage [21, 25, 28, 30]. Tailoring their properties requires a fundamental understanding of the intra- and intermolecular mechanisms governing their internal structure and dynamics. A puzzling observation, attributed to the bulk properties of concentrated electrolytes and ionic liquids, is the so-called underscreening, which is an anomalously large decay length of electrostatic interactions mediated by these liquids, as reported by recent surface force balance (SFB) experiments $[4,14,17,19,34]$.

For dilute electrolytes, the decay length is well described by the Debye-Hückel theory [12] 
with the Debye screening length

$$
\lambda_{\mathrm{D}}^{2}=\frac{\varepsilon_{0} \varepsilon_{r} k_{\mathrm{B}} T}{\sum_{i} \rho_{i} z_{i}^{2} e^{2}},
$$

where $\varepsilon_{0}$ is the vacuum permittivity, $\varepsilon_{r}$ the relative dielectric permittivity of a homogeneous background medium, $k_{\mathrm{B}}$ the Boltzmann constant, $T$ the absolute temperature, $\rho_{i}$ and $z_{i}$ the number density and the valency of species $i$, and $e$ the elementary charge. In the highconcentration regime, classical liquid state theories predict a damped oscillatory behavior, which is either core- or electrostatics-dominated, with the screening length exceeding $\lambda_{\mathrm{D}}$ and growing with increasing electrolyte concentration $[3,15,26,36]$. The aforementioned SFB experiments reported the emergence of a screening length $\lambda_{\mathrm{S}}$ roughly an order of magnitude larger than the ones predicted by classical theories [4, 14, 17, 19, 34]. In these experiments, the force was measured between two atomically flat, charged surfaces, confining neat ILs or electrolytes, and $\lambda_{\mathrm{S}}$ was extracted by fitting the force to a monotonic, exponentially decaying function of surface separation. All analyzed experimental data for $\lambda_{\mathrm{S}} v s$. ion concentration collapsed onto a single curve if appropriately rescaled. The corresponding scaling relation $[23,24]$

$$
\frac{\lambda_{\mathrm{S}}}{\lambda_{\mathrm{D}}} \propto\left(\frac{d}{\lambda_{\mathrm{D}}}\right)^{\alpha},
$$

where $d$ is the ion diameter and the scaling exponent $\alpha=3$, has been suggested to be a bulk property of concentrated electrolytes [23]. This behavior challenges our understanding of bulk ionic systems.

Herein, we report on long-range screening in selected bulk ionic systems obtained by extremely large-scale molecular dynamics (MD) simulations in volumes that encompass several of the experimentally measured screening lengths. As an example of a neat IL, we investigate 1-butyl-3-methylimidazolium hexafluorophosphate $\left(\left[\mathrm{C}_{4} \mathrm{C}_{1} \mathrm{Im}\right]^{+}\left[\mathrm{PF}_{6}\right]^{-}\right)$as there exist well-tested MD force fields for both all-atom and coarse-grained representations of this IL. We also perform all-atom simulations of aqueous sodium chloride $(\mathrm{NaCl})$ solutions with concentrations ranging from 1 to $5.2 \mathrm{~mol} / \mathrm{l}$. Finally, we conduct a series of simulations of 1-butyl-3-methylimidazolium bis(trifluoromethylsulfonyl)imide $\left(\left[\mathrm{C}_{4} \mathrm{C}_{1} \mathrm{Im}\right]^{+}\left[\mathrm{NTf}_{2}\right]^{-}\right)$in a racemic propylene carbonate $(\mathrm{PC})$ mixture (equal amounts of $(\mathrm{R})$ - and $(\mathrm{S})$-propylene carbonate) for IL mole fractions between $x=0.05$ and $x=1$ (pure $\left[\mathrm{C}_{4} \mathrm{C}_{1} \mathrm{Im}\right]^{+}\left[\mathrm{NTf}_{2}\right]^{-}$). This system is similar to the solution of 1-butyl-1-methylpyrrolidinium bis(trifluoromethylsulfonyl)imide $\left(\left[\mathrm{C}_{4} \mathrm{C}_{1} \mathrm{Pyrr}\right]^{+}\left[\mathrm{NTf}_{2}\right]^{-}\right)$in PC studied in Ref. 34 . All system compositions are listed in Sec. S1 
of the electronic supporting information (ESI).

Simulations have been performed with the GROMACS 2016.3 simulation package [1] in the $N p T$ ensemble using cubic boxes under periodic boundary conditions at temperature $T=300 \mathrm{~K}$ and pressure $p=1$ bar (simulation parameters are listed in Sec. S3 of the ESI). For ILs, we employed the $0.8^{*}$ OPLS-2009IL all-atom force field of (author?) [13] and the ILM2 force field of (author?) [31] for the coarse-grained description of $\left[\mathrm{C}_{4} \mathrm{C}_{1} \mathrm{Im}\right]^{+}\left[\mathrm{PF}_{6}\right]^{-}$. To simulate aqueous $\mathrm{NaCl}$ solutions, we used the KBFF ion parameters of (author?) [37] in conjunction with the extended simple point charge (SPC/E) water model [5]. To describe the $\mathrm{PC}$ interactions for the simulations of $\left[\mathrm{C}_{4} \mathrm{C}_{1} \mathrm{Im}\right]^{+}\left[\mathrm{NTf}_{2}\right]^{-}$in PC, we used the parameters provided by (author?) [35].

From the simulation trajectories, we computed radial distribution functions (RDFs) $g_{X Y}(r)$ ( $X$ and $Y$ denote ionic species). For our large-scale ionic systems, the computation of RDFs with GROMACS analysis tools would have taken several years to complete. We have therefore developed our own optimized analysis tool based on MDAnalysis [20, 27] and MPI for Python [9-11], allowing the evaluation of RDFs on hundreds of CPUs in parallel [38].

In spatially homogeneous systems, the effective interaction between ions $X$ and $Y$ is described by the potential of mean force (PMF), which is related to the RDF by

$$
w_{X Y}(r)=-k_{\mathrm{B}} T \ln \left(g_{X Y}(r)\right) .
$$

If the observed long-range decay $[4,14,17,19,34]$ is a property of bulk ionic liquids, one can expect the same asymptotic decay in an effective ion-ion interaction potential [22], and hence, in the corresponding PMF. Our further discussion will therefore be based on the analysis of PMFs.

Before discussing our results, it is important to stress that the experimentally observed transition between the damped oscillatory and the 'under-screened' monotonic regime occurred at separations between 4 and $7 \mathrm{~nm}$ for ILs, and up to $3 \mathrm{~nm}$ for $\mathrm{NaCl}[18,34]$. Thus, simulated systems have to be sufficiently large to allow the evaluation of PMFs far beyond these separations. Furthermore, ILs exhibit slow structural relaxations, necessitating simulation times of several hundred nanoseconds [16]. Unlike previous work [6], our simulations strictly fulfill both requirements. Since the magnitude of underscreening is expected to be small, possibly interfering with statistical errors, we also performed rigorous error analyses 
for all data series, taking temporal correlations into account [38].
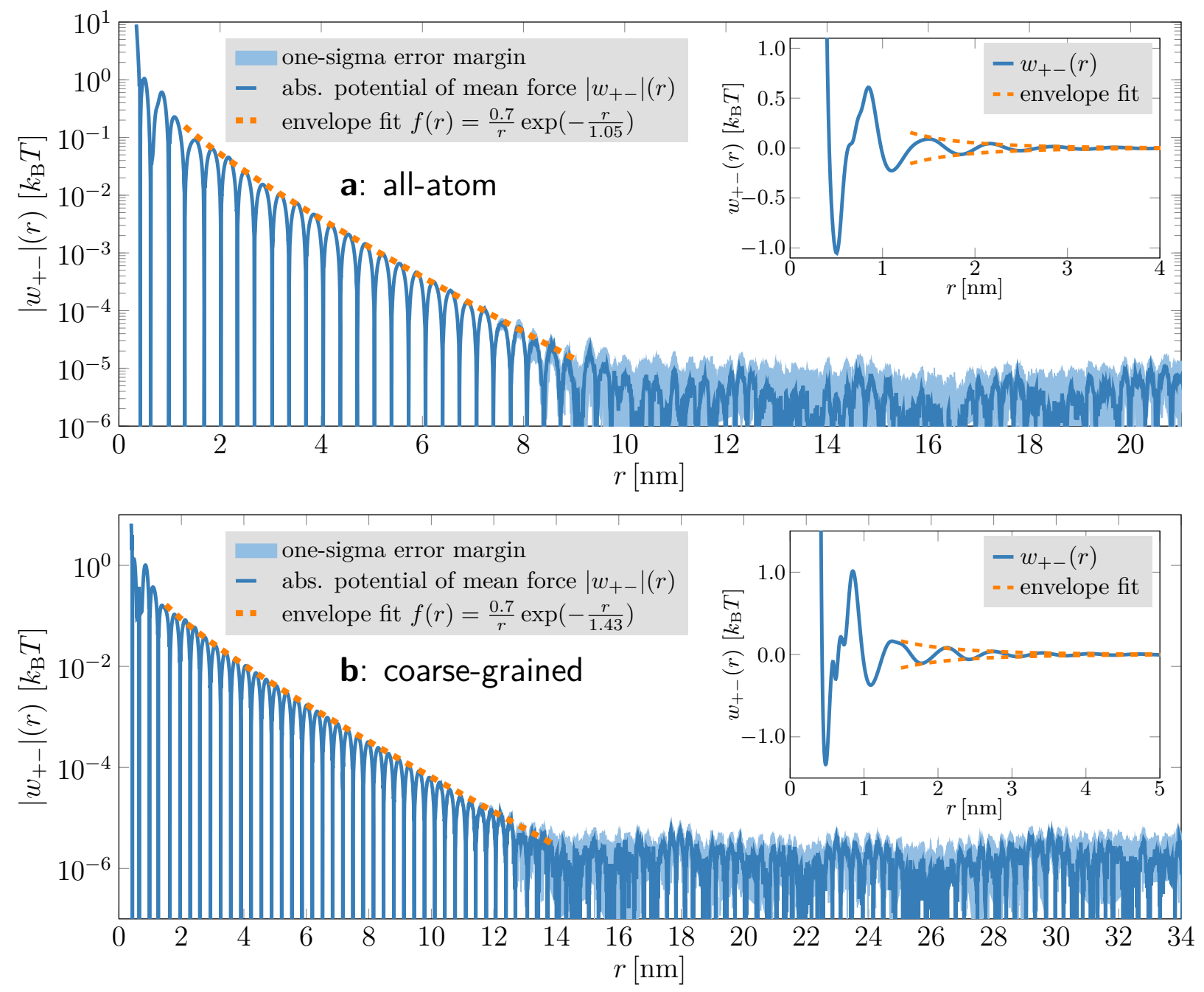

FIG. 1. Absolute value of the PMF $\left|w_{+-}\right|(r)$ between anions and cations (blue lines) in $\left[\mathrm{C}_{4} \mathrm{C}_{1} \mathrm{Im}\right]^{+}\left[\mathrm{PF}_{6}\right]^{-} .(\mathbf{a})$ : All-atom model. $w_{+-}(r)$ follows an oscillatory decay up to $r \approx 8.5 \mathrm{~nm}$, with the decay envelope (dashed, orange line) described by $f(r)=a / r \exp \left(-r / \lambda_{\mathrm{S}}\right)$ with $a=0.7 k_{\mathrm{B}} T$ and $\lambda_{\mathrm{S}}=1.05 \mathrm{~nm}$. For $r>8.5 \mathrm{~nm}$, the potential enters a region of almost constant noise level with rather high uncertainty (light blue area). Inset: The same anion-cation PMF $w_{+-}(r)$ with linear $y$-axis scaling. (b): Coarse-grained model. The qualitative features are the same as in the all-atom model, but the covered distances are up to $34 \mathrm{~nm}$. The extracted screening length $\lambda_{\mathrm{S}}=1.43 \mathrm{~nm}$ is larger than in the all-atom simulations due to its coarse-grained description.

For distances up to $r \approx 8.5 \mathrm{~nm}$, the PMF is well-described by an exponentially damped 
oscillatory hyperbolic decay

$$
\lim _{r \rightarrow \infty} w_{X Y}(r) \propto \frac{A}{r} \cos (k(r-\phi)) \exp \left(-\frac{r}{\lambda_{\mathrm{S}}}\right),
$$

where $A$ and $\phi$ are the amplitude and the phase shift, $\lambda_{\mathrm{S}}$ is the PMF's asymptotic decay length, and the wave vector $k$ determines the wavelength of its oscillation. The envelope of this decay is indicated in Fig. 1a by a dashed orange line (we excluded the region $r<1.3 \mathrm{~nm}$ from the analysis because it is strongly affected by short-ranged Lennard-Jones interactions). The extracted decay length $\lambda_{\mathrm{S}}=1.05 \mathrm{~nm}$ is consistent with classical theories $[3,15,26,36]$. The cation-cation and anion-anion PMFs exhibit a similar behavior with the same decay length [38]. The statistical uncertainty is quite high for $r \gtrsim 8.5 \mathrm{~nm}$ and a hypothetical monotonic decay in this region might be hidden in the noise. However, experiments with similar ILs [34] suggest an onset of the monotonic decay already at smaller separations, which is not present in our data.

In coarse-grained simulations of $\left[\mathrm{C}_{4} \mathrm{C}_{1} \mathrm{Im}\right]^{+}\left[\mathrm{PF}_{6}\right]^{-}$, the box edge length was almost $50 \mathrm{~nm}$ and the system comprised 358296 ion pairs (1 433184 interaction sites). The PMFs were calculated for distances up to $34 \mathrm{~nm}$ (Fig. 1b). They exhibit the same qualitative behavior as the all-atom model, but yield a larger decay length $\lambda_{\mathrm{S}}=1.43 \mathrm{~nm}$, which can be attributed to the coarse-grained description. Due to the larger number of ions in the system, the increased statistical accuracy allowed us to resolve the oscillatory decay at distances up to $13 \mathrm{~nm}$. For larger distances, the PMF again enters a region of almost constant noise level.

Next, we analyze a $4.43 \mathrm{~mol} / \mathrm{l}$ aqueous $\mathrm{NaCl}$ solution. This system comprised 216000 ion pairs and 2458296 water molecules (7806 888 atoms in total) in a simulation box with an edge length of $43.25 \mathrm{~nm}$. The cation-anion PMF obtained from a $200 \mathrm{~ns}$ simulation run is displayed in Fig. 2. Although in this case a superposition of several damped oscillatory functions was needed to fit the PMFs (cf. Eq. (5)), up to $2.2 \mathrm{~nm}$ their envelope could still be approximated by a single decay (dashed orange line in Fig. 2). The extracted decay length $\lambda_{\mathrm{S}}=0.2 \mathrm{~nm}$ is again consistent with classical theories [3, 15, 26, 36]. For distances exceeding $2.2 \mathrm{~nm}$, the PMF becomes very noisy and no distinct oscillations are discernible. In this region, the PMF's envelope may seem to follow a long-ranged decay. However, this decay is entirely due to statistical noise, which decreases with distance. It can be shown that the noise in the PMF of an ideal gas, comprising the same number of particles in the same volume, exhibits the very same decay [38]. Hence, in this case, there is also no 
anomalously long-ranged monotonic decay of interionic interactions detectable within an accuracy of $\approx 10^{-5} k_{\mathrm{B}} T$.

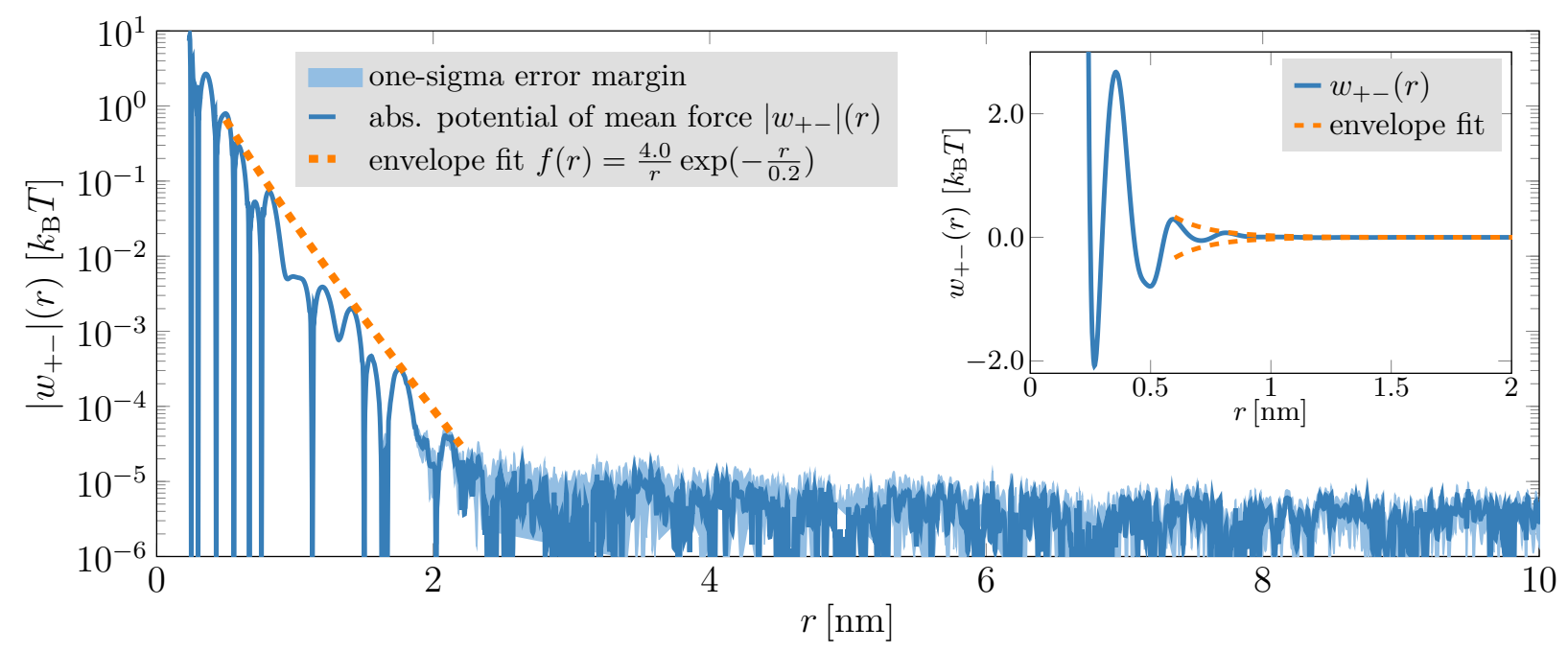

FIG. 2. Absolute value of the PMF $\left|w_{+-}\right|(r)$ between anions and cations (blue line) in a $4.43 \mathrm{~mol} / \mathrm{l}$ aqueous $\mathrm{NaCl}$ solution. Up to a distance of about $2.2 \mathrm{~nm}, w_{+-}(r)$ exhibits an oscillatory decay which is comprised of a superposition of several oscillations with different parameters. Nevertheless, the envelope of the decay (dashed, orange line) can be approximated by $f(r)=a / r \exp \left(-r / \lambda_{\mathrm{S}}\right)$ with amplitude $a=4.0 k_{\mathrm{B}} T$ and decay length $\lambda_{\mathrm{S}}=0.2 \mathrm{~nm}$. For distances larger than $2.2 \mathrm{~nm}$, the potential lies in the order of the uncertainty level of about $10^{-5} k_{\mathrm{B}} T$. Its further decay is not a feature of the system but simply due to the statistical error, which decreases with increasing distance.

To study the concentration dependence of the screening lengths, we simulated aqueous $\mathrm{NaCl}$ electrolytes for ion concentrations ranging from 1.16 to $5.19 \mathrm{~mol} / \mathrm{l}$. Each system comprised 3750 ion pairs and a concentration-dependent number of water molecules. In addition, we conducted all-atom simulations of $\left[\mathrm{C}_{4} \mathrm{C}_{1} \mathrm{Im}\right]^{+}\left[\mathrm{NTf}_{2}\right]^{-}$in $\mathrm{PC}$ at various concentrations. As there has been no sign of anomalously large screening lengths, we chose to use smaller simulation boxes in favor of covering a larger number of IL concentrations. With the exception of the two lowest concentrations, all systems contained 500 ion pairs and a suitably adjusted number of PC molecules. For each system, we performed up to four independent simulation runs with more than $1 \mu$ s per run.

Unlike for neat $\left[\mathrm{C}_{4} \mathrm{C}_{1} \mathrm{Im}\right]^{+}\left[\mathrm{PF}_{6}\right]^{-}$, these systems required a superposition of several os- 
cillatory exponentially damped hyperbolas

$$
f(r)=\sum_{n=1}^{k} \frac{A_{n}}{r} \cos \left(\omega_{n} r-\phi_{n}\right) \exp \left(-\frac{r}{\lambda_{n}}\right)
$$

to fit the data. We found that $k=2$ was sufficient to obtain an excellent fit for all IL mixtures in the range $1.2 \leq r \leq 3 \mathrm{~nm}$. The PMFs of aqueous $\mathrm{NaCl}$ solutions required $k=3$ to fit in the range $0.8 \leq r \leq 2 \mathrm{~nm}$ (for fit parameters see Sections S2.4 and S2.5 of the ESI). Figure 3 shows that the obtained screening lengths $\lambda_{\mathrm{S}}=\max \left(\lambda_{n}\right)$ are almost an order of magnitude smaller than those measured in SFB experiments of similar systems [34].

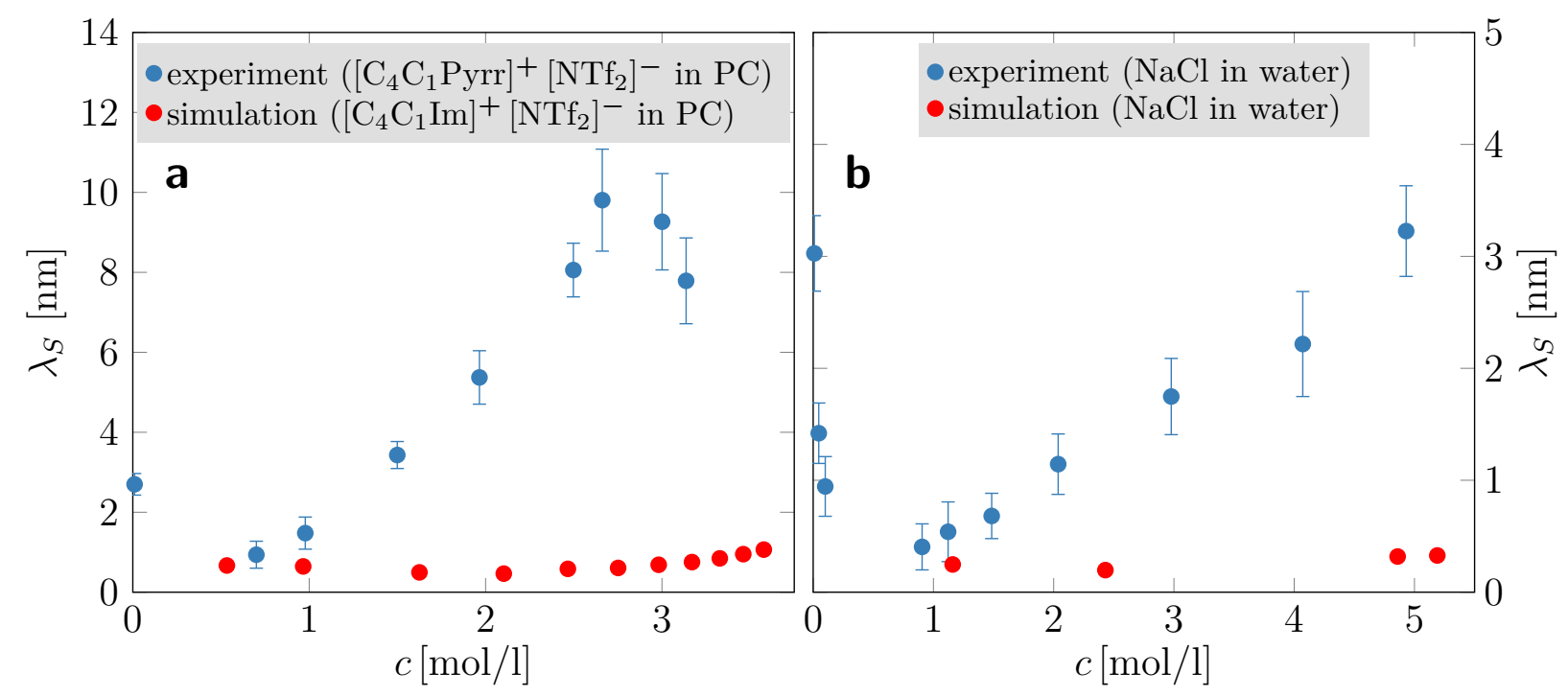

FIG. 3. Concentration-dependent screening lengths $\lambda_{\mathrm{S}}$ obtained from MD simulations compared to those obtained from experimental SFB measurements for similar ionic species. (a): Experimentally determined electrostatic screening lengths of $\left[\mathrm{C}_{4} \mathrm{C}_{1} \mathrm{Pyrr}\right]^{+}\left[\mathrm{NTf}_{2}\right]^{-}$in PC (blue dots) compared to simulation results of $\left[\mathrm{C}_{4} \mathrm{C}_{1} \mathrm{Im}\right]^{+}\left[\mathrm{NTf}_{2}\right]^{-}$in $\mathrm{PC}$ (red dots). (b): Screening lengths of aqueous $\mathrm{NaCl}$ solutions (experiment: blue dots; simulation: red dots). Experimental data are taken from Ref. 34 .

Determining scaling relations for the correlation lengths, akin to Eq. (2), requires the knowledge of an average ion diameter $d$ and the Debye length $\lambda_{\mathrm{D}}$. For $\left[\mathrm{C}_{4} \mathrm{C}_{1} \operatorname{Im}\right]^{+}\left[\mathrm{NTf}_{2}\right]^{-}$, we used the number of ion pairs $N_{\text {IP }}$ and the simulation box volume $V$ to determine $d=\frac{1}{2}\left(V / N_{\mathrm{IP}}\right)^{\frac{1}{3}} \approx 0.39 \mathrm{~nm} ;$ for $\mathrm{NaCl}$ we took $d=0.294 \mathrm{~nm}$ from Ref. 34 . Calculating $\lambda_{\mathrm{D}}$ using Eq. (1) requires the knowledge of the static relative dielectric permittivity $\epsilon_{r}$ of a background medium. We computed $\varepsilon_{r}$ of the entire system, as in Ref. 23, by using the Einstein-Helfand method [32]. The obtained values of $\varepsilon_{r}$ compare well with the available 
experimental data for pure PC [7] and pure $\left[\mathrm{C}_{4} \mathrm{C}_{1} \mathrm{Im}\right]^{+}\left[\mathrm{NTf}_{2}\right]^{-}[8]$. For aqueous $\mathrm{NaCl}$, our $\epsilon_{r}$ is systematically lower than the experimental values, due to the SPC/E model underestimating the permittivity of water [33]. Nevertheless, the concentration-dependent trend is well reproduced (cf. Ref. 29). The values of $\epsilon_{r}$ are listed in Sec. S2.2 of the ESI.
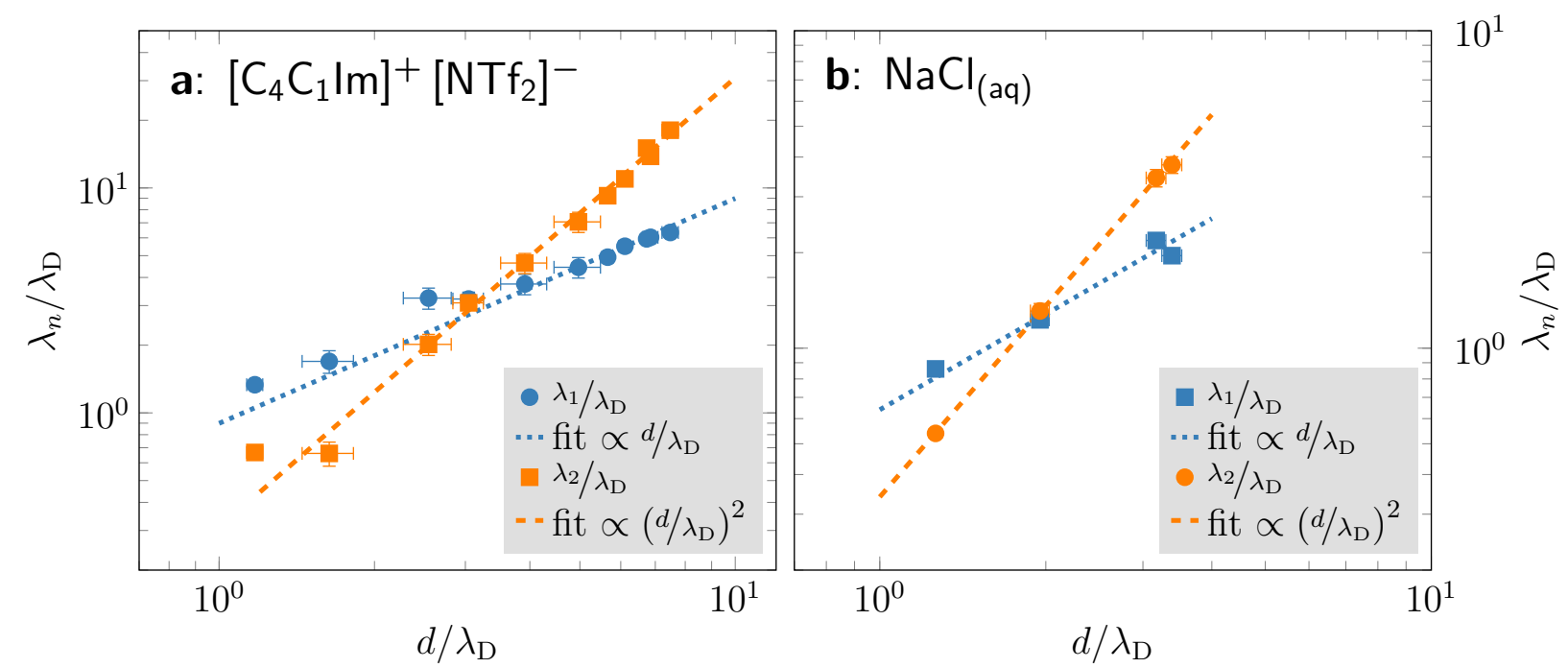

FIG. 4. Scaling of the concentration-dependent screening lengths of $\left[\mathrm{C}_{4} \mathrm{C}_{1} \mathrm{Im}\right]^{+}\left[\mathrm{NTf}_{2}\right]^{-}$in propylene carbonate (left) and $\mathrm{NaCl}$ in water (right) as determined by fits of Eq. (5) to cation-anion PMFs $w_{+-}(r)$. The ratio of the screening $\lambda_{n}$ to the concentration-dependent Debye length $\lambda_{\mathrm{D}}$ is shown as a function of the average ion diameter $d$ divided by $\lambda_{\mathrm{D}}$. For both substances, we see two decay lengths, each corresponding to a distinct wavelength $2 \pi / \omega_{n}$, and both exhibiting a power law dependence. For both systems, the asymptotic decay length ratio for high concentrations $\lambda_{2} / \lambda_{\mathrm{D}}$ is proportional to $\left(d / \lambda_{\mathrm{D}}\right)^{2}$.

Figure 4 shows the ratio $\lambda_{n} / \lambda_{\mathrm{D}}(n=1,2)$ as a function of $d / \lambda_{\mathrm{D}}$ with $\lambda_{n}$ obtained from fitting the PMFs to Eq. (5). The asymptotic linear and quadratic scaling

$$
\frac{\lambda_{n}}{\lambda_{\mathrm{D}}} \propto\left(\frac{d}{\lambda_{\mathrm{D}}}\right)^{n}
$$

is clearly visible in Fig. 4. Interestingly, the two screening lengths cross each other, so that the screening length with the quadratic scaling prevails at high concentrations[2].

In conclusion, our extremely large-scale MD simulations of concentrated electrolytes and neat ionic liquids allowed us to calculate interionic PMFs with unprecedented precision and to analyze their behavior in a range of distances relevant to experiments $[4,14,17,19,34]$. 
We revealed the existence of two screening lengths showing linear and quadratic scaling, Eq. (6). However, within a PMF accuracy of $10^{-5} k_{\mathrm{B}} T$, we observed no evidence for an anomalously long-ranged, monotonic decay in effective ionic interactions. These results demonstrate that underscreening is unlikely an equilibrium bulk property of concentrated ionic systems.

We acknowledge support by the High Performance and Cloud Computing Group at the Zentrum für Datenverarbeitung of the University of Tübingen, the state of BadenWürttemberg through bwHPC and the German Research Foundation (DFG) through grant no INST 37/935-1 FUGG. We are grateful for computing resources on the Cray XC40 (Hazel Hen) cluster provided by the High Performance Computing Center Stuttgart (HLRS), Germany. C. H. acknowledges financial contributions from the Deutsche Forschungsgemeinschaft (DFG, German Research Foundation) under Germany's Excellence Strategy - EXC 2075 390740016.

* E-mail: holm@icp.uni-stuttgart.de

[1] Mark James Abraham, Teemu Murtola, Roland Schulz, Szilárd Páll, Jeremy C Smith, Berk Hess, and Erik Lindahl. GROMACS: High performance molecular simulations through multilevel parallelism from laptops to supercomputers. SoftwareX, 1:19-25, 2015. doi:10.1016/ j.softx.2015.06.001.

[2] Ram M. Adar, Samuel A. Safran, Haim Diamant, and David Andelman. Screening length for finite-size ions in concentrated electrolytes. Physical Review E, 100:042615, 2019. doi: 10.1103/physreve.100.042615.

[3] Phil Attard. Asymptotic analysis of primitive model electrolytes and the electrical double layer. Physical Review E, 48:3604-3621, 1993. doi:10.1103/physreve.48.3604.

[4] Theodoros Baimpos, Buddha R. Shrestha, Sangeetha Raman, and Markus Valtiner. Effect of interfacial ion structuring on range and magnitude of electric double layer, hydration, and adhesive interactions between mica surfaces in $0.05-3 \mathrm{M} \mathrm{Li}^{+}$and $\mathrm{Cs}^{+}$electrolyte solutions. Langmuir, 30(15):4322-4332, 2014. doi:10.1021/la500288w. 
[5] H. J. C. Berendsen, J. R. Grigera, and T. P. Straatsma. The missing term in effective pair potentials. The Journal of Physical Chemistry, 91(24):6269-6271, November 1987. doi: 10.1021/j100308a038.

[6] Samuel W. Coles, Chanbum Park, Rohit Nikam, Matej Kanduč, Joachim Dzubiella, and Benjamin Rotenberg. Correlation length in concentrated electrolytes: Insights from all-atom molecular dynamics simulations. The Journal of Physical Chemistry B, 124(9):1778-1786, 2020. doi:10.1021/acs.jpcb.9b10542.

[7] Jean-François Côté, Dany Brouillette, Jacques E. Desnoyers, Jean-François Rouleau, JeanMarie St-Arnaud, and Gérald Perron. Dielectric constants of acetonitrile, $\gamma$-butyrolactone, propylene carbonate, and 1,2-dimethoxyethane as a function of pressure and temperature. Journal of Solution Chemistry, 25(12):1163-1173, 1996. doi:10.1007/BF00972644.

[8] Corinne Daguenet, Paul J. Dyson, Ingo Krossing, Alla Oleinikova, John Slattery, Chihiro Wakai, and Hermann Weingärtner. Dielectric response of imidazolium-based roomtemperature ionic liquids. The Journal of Physical Chemistry B, 110(25):12682-12688, 2006. doi:10.1021/jp0604903.

[9] Lisandro D. Dalcín, Rodrigo R. Paz, Pablo A. Kler, and Alejandro Cosimo. Parallel distributed computing using Python. Advances in Water Resources, 34(9):1124-1139, 2011. doi:10. 1016/j . advwatres.2011.04.013.

[10] Lisandro D. Dalcín, Rodrigo R. Paz, and Mario Storti. MPI for Python. Journal of Parallel and Distributed Computing, 65(9):1108-1115, 2005. doi:10.1016/j.jpdc.2005.03.010.

[11] Lisandro D. Dalcín, Rodrigo R. Paz, Mario Storti, and Jorge D’Elía. MPI for Python: Performance improvements and MPI-2 extensions. Journal of Parallel and Distributed Computing, 68(5):655-662, 2008. doi:10.1016/j.jpdc.2007.09.005.

[12] P. Debye and E. Hückel. Zur Theorie der Elektrolyte. I. Gefrierpunktserniedrigung und verwandte Erscheinungen. Physikalische Zeitschrift, 24(9):185-206, 1923.

[13] Brian Doherty, Xiang Zhong, Symon Gathiaka, Bin Li, and Orlando Acevedo. Revisiting OPLS force field parameters for ionic liquid simulations. Journal of Chemical Theory and Computation, 13(12):6131-6145, 2017. doi:10.1021/acs.jctc.7b00520.

[14] R. M. Espinosa-Marzal, A. Arcifa, A. Rossi, and N. D. Spencer. Microslips to "avalanches" in confined, molecular layers of ionic liquids. The Journal of Physical Chemistry Letters, 5(1):179-184, 2014. doi:10.1021/jz402451v. 
[15] R. Evans, R. J. F. Leote de Carvalho, J. R. Henderson, and D. C. Hoyle. Asymptotic decay of correlations in liquids and their mixtures. The Journal of Chemical Physics, 100(1):591-603, 1994. doi:10.1063/1.466920.

[16] Sonja Gabl, Christian Schröder, and Othmar Steinhauser. Computational studies of ionic liquids: Size does matter and time too. The Journal of Chemical Physics, 137(9):094501, 2012. doi:10.1063/1.4748352.

[17] Matthew A. Gebbie, Howard A. Dobbs, Markus Valtiner, and Jacob N. Israelachvili. Longrange electrostatic screening in ionic liquids. Proceedings of the National Academy of Sciences of the United States of America, 112(24):7432-7437, 2015. doi:10.1073/pnas.1508366112.

[18] Matthew A. Gebbie, Alexander M. Smith, Howard A. Dobbs, Alpha A. Lee, Gregory G. Warr, Xavier Banquy, Markus Valtiner, Mark W. Rutland, Jacob N. Israelachvili, Susan Perkin, and Rob Atkin. Long range electrostatic forces in ionic liquids. Chemical Communications, 53:1214-1224, 2017. doi:10.1039/c6cc08820a.

[19] Matthew A. Gebbie, Markus Valtiner, Xavier Banquy, Eric T. Fox, Wesley A. Henderson, and Jacob N. Israelachvili. Ionic liquids behave as dilute electrolyte solutions. Proceedings of the National Academy of Sciences of the United States of America, 110(24):9674-9679, 2013. doi:10.1073/pnas.1307871110.

[20] Richard J. Gowers, Max Linke, Jonathan Barnoud, Tyler J. E. Reddy, Manuel N. Melo, Sean L. Seyler, Jan Domański, David L. Dotson, Sébastien Buchoux, Ian M. Kenney, and Oliver Beckstein. MDAnalysis: A Python package for the rapid analysis of molecular dynamics simulations. In Sebastian Benthall and Scott Rostrup, editors, Proceedings of the 15th Python in Science Conference, pages 98-105, 2016. doi:10.25080/Majora-629e541a-00e.

[21] Robert Hayes, Gregory G. Warr, and Rob Atkin. Structure and nanostructure in ionic liquids. Chemical Reviews, 115(13):6357-6426, 2015. doi:10.1021/cr500411q.

[22] Roland Kjellander. Focus article: Oscillatory and long-range monotonic exponential decays of electrostatic interactions in ionic liquids and other electrolytes: The significance of dielectric permittivity and renormalized charges. The Journal of Chemical Physics, 148(19):193701, 2018. doi:10.1063/1.5010024.

[23] Alpha A. Lee, Carla S. Perez-Martinez, Alexander M. Smith, and Susan Perkin. Scaling analysis of the screening length in concentrated electrolytes. Physical Review Letters, 119:026002, 2017. doi:10.1103/PhysRevLett.119.026002. 
[24] Alpha A. Lee, Carla S. Perez-Martinez, Alexander M. Smith, and Susan Perkin. Underscreening in concentrated electrolytes. Faraday Discussions, 199:239-259, 2017. doi: 10.1039/c6fd00250a.

[25] Zhigang Lei, Biaohua Chen, Yoon-Mo Koo, and Douglas R. MacFarlane. Introduction: Ionic liquids. Chemical Reviews, 117(10):6633-6635, 2017. doi:10.1021/acs .chemrev.7b00246.

[26] R. J. F. Leote de Carvalho and R. Evans. The decay of correlations in ionic fluids. Molecular Physics, 83(4):619-654, 1994. doi:10.1080/00268979400101491.

[27] Naveen Michaud-Agrawal, Elizabeth J. Denning, Thomas B. Woolf, and Oliver Beckstein. MDAnalysis: A toolkit for the analysis of molecular dynamics simulations. Journal of Computational Chemistry, 32(10):2319-2327, 2011. doi:10.1002/jcc.21787.

[28] Siddharth Pandey. Analytical applications of room-temperature ionic liquids: A review of recent efforts. Analytica Chimica Acta, 556(1):38-45, 2006. doi:10.1016/j.aca.2005.06. 038.

[29] A. Peyman, C. Gabriel, and E. H. Grant. Complex permittivity of sodium chloride solutions at microwave frequencies. Bioelectromagnetics, 28(4):264-274, 2007. doi:10.1002/bem. 20271.

[30] Robin D. Rogers and Kenneth R. Seddon. Ionic liquids-solvents of the future? Science, 302(5646):792-793, 2003. doi:10.1126/science.1090313.

[31] Durba Roy and Mark Maroncelli. An improved four-site ionic liquid model. Journal of Physical Chemistry B, 114(39):12629-12631, 2010. doi:10.1021/jp108179n.

[32] C. Schröder, M. Haberler, and O. Steinhauser. On the computation and contribution of conductivity in molecular ionic liquids. The Journal of Chemical Physics, 128(13):134501, 2008. doi:10.1063/1.2868752.

[33] Marcello Sega and Christian Schröder. Dielectric and terahertz spectroscopy of polarizable and nonpolarizable water models: A comparative study. The Journal of Physical Chemistry A, 119(9):1539-1547, 2015. doi:10.1021/jp507419e.

[34] Alexander M. Smith, Alpha A. Lee, and Susan Perkin. The electrostatic screening length in concentrated electrolytes increases with concentration. The Journal of Physical Chemistry Letters, 7(12):2157-2163, 2016. doi:10.1021/acs.jpclett.6b00867.

[35] Munetaka Takeuchi, Yasuo Kameda, Yasuhiro Umebayashi, Sari Ogawa, Takaaki Sonoda, Shin-ichi Ishiguro, Miho Fujita, and Mitsuru Sano. Ion-ion interactions of $\mathrm{LiPF}_{6}$ and $\mathrm{LiBF}_{4}$ in propylene carbonate solutions. Journal of Molecular Liquids, 148(2):99-108, 2009. doi: 
10.1016/j.molliq.2009.07.003.

[36] Luis M. Varela, Manuel García, and Víctor Mosquera. Exact mean-field theory of ionic solutions: non-Debye screening. Physics Reports, 382(1):1-111, 2003. doi:10.1016/ s0370-1573(03)00210-2.

[37] Samantha Weerasinghe and Paul E. Smith. A Kirkwood-Buff derived force field for sodium chloride in water. The Journal of Chemical Physics, 119(21):11342-11349, December 2003. doi:10.1063/1.1622372.

[38] Johannes Zeman, Svyatoslav Kondrat, and Christian Holm. To be published. 